\title{
Microfluidic fuel cells on paper: meeting the power needs of next generation lateral flow devices $\uparrow$
}

Cite this: Energy Environ. Sci., 2014, 7, 1744

Received 13th December 2013 Accepted 12th February 2014

DOI: $10.1039 / c 3 e e 44044 c$

www.rsc.org/ees

\author{
J. P. Esquivel, ${ }^{\star a b}$ F. J. Del Campo, ${ }^{a}$ J. L. Gómez de la Fuente, ${ }^{c}$ S. Rojas ${ }^{c}$ and N. Sabaté ${ }^{a}$
}

Lateral flow test strips have dominated the rapid diagnostics landscape for decades. Recently, the emergence of paper microfluidics has brought new functionalities to these porous materials, and the search for instrument-free point-of-care devices has driven the development of different types of energy sources to fulfill their power needs. This work presents the development of microfluidic fuel cells as paper-based power sources in a standard lateral flow test format. These fuel cells benefit from the laminar flow occurring in a porous material by capillarity to separately react with two parallel streams, anolyte and catholyte, without an ionic exchange membrane or external pumps. It has been shown that the devices are capable of delivering power densities in the range of $1-5 \mathrm{~mW} \mathrm{~cm}^{-2}$ using solutions of methanol and $\mathrm{KOH}$. The incorporation of a conjugate pad to store the $\mathrm{KOH}$ electrolyte in a solid form and a methanol-rich agar gel on top of the reaction membrane allows the fuel cell to function soaking a single sample pad with just water. The presented microfluidic fuel cell approach would enable a more straightforward integration with typical lateral flow test strips and a cost-effective manufacturing. This work represents the starting point in the development of a power source for capillary-based autonomous sensing systems capable of harvesting the energy needed for the measurement from the biological sample to be analyzed.

\section{Broader context}

Lateral flow tests strips have been extremely successful in the diagnostics field because of their low-cost, lightweight, portability and ability to yield a rapid and easy-to-read answer. The capabilities of these devices have recently been rediscovered and are now being reconfigured to create new and exciting components such as valves, mixers, separators or displays, where the fluids are driven by capillary action and no external pressure sources, e.g. pumps, are needed. The addition of functionalities within a paper matrix empowers the vision of the ideal paper-based microfluidic device that will perform complex analytical functions with minimal effort and manipulation by the user, who would just add the sample to the system and then interpret the readout. The approach presented in this work combines the advantages of microfluidic fuel cells and the convenience and simplicity of lateral flow test strips to bring closer that vision. Ultimately, the unique electrochemical features of fuel cells make them capable to generate the power needed to quantify the results of a rapid flow test from the same sample that is analysed (e.g. glucose in blood or urea in urine) and in this way achieve the integration of an autonomous paper-based sensing system.

\section{Introduction}

The capabilities of paper have recently been rediscovered and some of its intrinsic properties such as capillarity, porosity, surface area or patternability are being used to develop complex microfluidic functions. ${ }^{1}$ Materials used in lateral

${ }^{a}$ Instituto de Microelectrónica de Barcelona, IMB-CNM (CSIC), Campus UAB sn, Bellaterra, Barcelona 08193, Spain. E-mail: juanpablo.esquivel@csic.es

${ }^{b}$ Department of Bioengineering, University of Washington, Seattle, Washington 98195 , United States. E-mail: juanpa@uw.edu

'Instituto de Catálisis y Petroleoquímica, ICP (CSIC), Madrid 28049, Spain

$\dagger$ Electronic supplementary information (ESI) available: Additional figures and videos for the visualization of flows using color dyes, details on electrochemical characterization of parallel laminar flows of two solutions, fuel cell partial potentials, fuel cell reproducibility and comparison of microfluidic methanol fuel cells. See DOI: 10.1039/c3ee44044c flow devices are now being adapted and reconfigured to create new and exciting components such as valves, mixers, separators or displays, where the fluids are driven by capillary action and no external pressure sources, e.g. pumps, are needed. Ultimately, the addition of functionalities within a paper matrix empowers the vision of the ideal paper-based microfluidic device that will perform complex analytical functions with minimal effort and manipulation by the user, who would just add the sample to the system and then interpret the readout. In this path towards simplicity, dedicated readers are now being substituted by a camera smartphone or implemented on-chip. ${ }^{2}$ The Clearblue Digital Pregnancy Test (Swiss Precision Diagnostics $\mathrm{GmbH})^{3}$ is a fully disposable lateral flow test that relies on a small button-cell battery to perform an optical measurement and yield semiquantitative results. Despite its commercial success, this 
approximation is rather blunt: after a single use, the whole device is thrown away. This includes the lateral flow strip, the plastic casing, the optical sensor, an LCD display, electronic components mounted on a PCB, and an almost fully charged battery. A much more compact and environmentally friendly on-chip solution has been recently presented by Acreo Swedish ICT AB (Kista, Sweden). The device uses the advantages of printed electronics to perform a completely disposable amperometric test that includes a sensor, electronics and a printed battery in a small card format. ${ }^{4}$ It is important to stress that the availability of an on-board energy source that is easy to dispose of and compatible with paper technology is crucial to realize this vision. This has brought about the search for a new generation of power sources that satisfy the requirements such as high power density, low cost and disposability with minimum environmental impact. Particularly relevant are the studies by Nyholm $e t$ al..$^{5}$ describing the use of electrochemical supercapacitors, the fluidic batteries described by Thom et al., ${ }^{6}$ and the biofuel cells reported by Zhang et al. ${ }^{7}$ and Ciniciato et al. ${ }^{8}$ Moreover, a recent review article summarizes and examines these energy generation and storage approaches available in the literature. ${ }^{9}$

In this work we focus on fuel cells as very suitable candidates to supply on-board energy to the next generation of lateral flow-based devices. Fuel cells convert chemical energy into electrical energy. Their high energy density together with the possibility to operate at room temperature using liquid fuels identified them as potentially suitable for portable applications more than a decade ago. Despite their promising capabilities, they have failed to oust lithium batteries from the consumer electronics market: batteries have successfully evolved into tiny packages that are very easy to use, rechargeable and that can meet the power demands of current portable appliances. In contrast, fuel cells have to be fed with a liquid fuel and require an ion-conducting membrane that prevents mixing of oxidant and reducing species, which has to be permanently hydrated to ensure optimal performance. The complexity associated with the use of this membrane was overcome some years ago with the development of the so-called microfluidic fuel cells, ${ }^{\mathbf{1 0}-12}$ in which the separation of reactants is achieved by forcing them to flow in parallel in a microfluidic channel at low Reynolds numbers. Microfluidic fuel cells yield significant amounts of energy with a very simple architecture. However, most still require the use of external pumps to keep reactants in motion, which limits severely their portability and miniaturization.

In this work we show that when implemented in paper, microfluidic fuel cells benefit from capillarity as reactants flow without external assistance. As they need a flowing fluid, they can benefit from the use of materials and processes already used by the lateral flow test industry which at the same time makes its integration within a same lateral flow test very straightforward. Thus, this new portable power source combines the advantages of microfluidic fuel cells and the convenience and simplicity of lateral flow test strips.

\section{Design and working principle}

A microfluidic fuel cell is defined as a fuel cell in which the anolyte and catholyte flow side-by-side through a microfluidic channel at a low Reynolds number (i.e. laminar flow). Under this regime, mixing of the two streams occurs only by diffusion and is restricted to an interfacial width at the center of the channel. Thus, if the anode and cathode are placed at sufficient distance from the inter-diffusion zone, electrode reactions take place independently. Ionic transport within the streams is achieved by diluting a supporting electrolyte and electrical power is extracted by connecting both electrodes to an external load. The width of the reactants mixing zone can be controlled by modification of channel dimensions and flow rates, so as to ensure a proper functioning of the device it is crucial to ensure the continuous flow of reagents.

The implementation of a fuel cell within a paper strip allows establishment of a flow without the use of external pumps. Moreover, the laminar flow is guaranteed in paper since the relevant length scale is the membrane pore diameter (typically from 1 to $10 \mu \mathrm{m}$ ) resulting in Reynolds numbers on the order of $10^{-3}$ at typical lateral flow rates. Indeed, in 2010, Osborn et al. showed that two solutions could flow in parallel without convective mixing in a single paper strip driven only by capillary forces. ${ }^{13}$ Hence, building on our previous experience, ${ }^{14}$ we set out to develop a paper-based microfluidic fuel cell.

Beyond demonstrating the intrinsic advantages of building this device in paper-based materials, our goal was to make these fuel cells compatible with the manufacturing processes used in the fabrication of lateral flow test strips. Therefore the device is similar in its construction to a typical lateral flow strip, i.e. it comprises sample pad, conjugate pad, reaction membrane, and absorbent pad. Anode and cathode electrodes have been placed at the bottom and top of the reaction zone in the main paper strip. Fig. 1 shows a schematic representation of the reaction zone in our paper microfluidic fuel cell. Due to its high energy density and well-known catalysis, methanol has been chosen as a fuel. $\mathrm{KOH}$ was the electrolyte used to maintain an alkaline media and enhance the methanol oxidation reaction at the anode. The electrochemical processes taking place in this fuel cell are: ${ }^{15}$

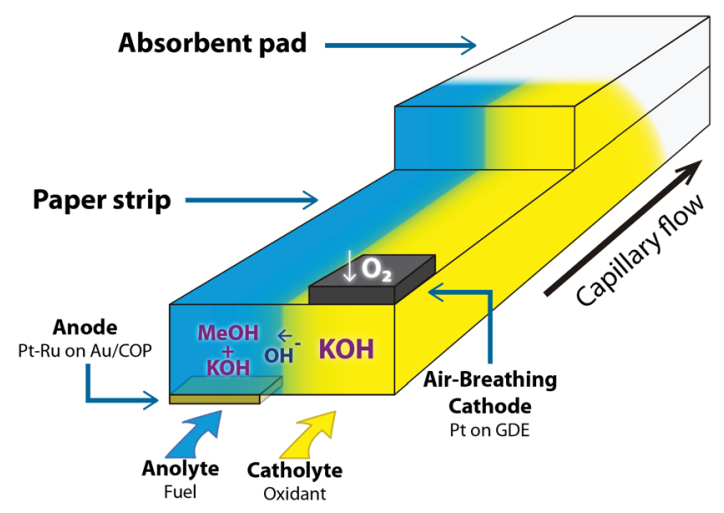

Fig. 1 Schematic of a paper-based microfluidic fuel cell design. 
Anode:

$$
\mathrm{CH}_{3} \mathrm{OH}+6 \mathrm{OH}^{-} \rightarrow \mathrm{CO}_{2}+5 \mathrm{H}_{2} \mathrm{O}+6 \mathrm{e}^{-}, E^{0}=-0.81 \mathrm{~V}
$$

Cathode:

$$
\frac{3}{2} \mathrm{O}_{2}+3 \mathrm{H}_{2} \mathrm{O}+6 \mathrm{e}^{-} \rightarrow 6 \mathrm{OH}^{-}, E^{0}=0.40 \mathrm{~V}
$$

Overall:

$$
\mathrm{CH}_{3} \mathrm{OH}+\frac{3}{2} \mathrm{O}_{2} \rightarrow \mathrm{CO}_{2}+2 \mathrm{H}_{2} \mathrm{O}, \Delta E=1.21 \mathrm{~V}
$$

In order to establish two parallel flows, the basic design of the lateral flow strip has to be modified. Here we present an incremental approach. First, we demonstrate a lateral flow device with two differentiated but confluent sample pads for anolyte and catholyte. This fuel cell is used in this paper to prove the concept and perform systematic characterization at different fuel and electrolyte concentrations. Second, we show how the incorporation of a conjugate pad to store the $\mathrm{KOH}$ electrolyte in a solid form and a piece of a methanol-rich agar gel on top of the reaction membrane allows the fuel cell to function soaking a single sample pad with just water.

Our first design, depicted in Fig. 2a, is based on the "Y" architecture typically used in the first published approaches to microfluidic fuel cells. In this case, it consisted of a $5 \mathrm{~mm}$ wide paper strip (Fusion 5, Whatman) divided at one end into two $2 \mathrm{~mm}$ wide inlets connected to larger pads that were soaked with the reactants. At the other end of the paper strip, the membrane was covered by an absorbent pad consisting of two additional layers $(5 \times 25 \mathrm{~mm})$ of the same porous material. Together with the membrane, this end of the strip could store more than 200 $\mu \mathrm{L}$ of liquid. The anode electrode consisted of a thin Au layer evaporated on a $4 \times 5 \mathrm{~mm}$ COP (Zeonor ZF14-188) piece, while a $4 \times 5 \mathrm{~mm}$ piece of carbon paper (Toray EC-TP1-060T) was used

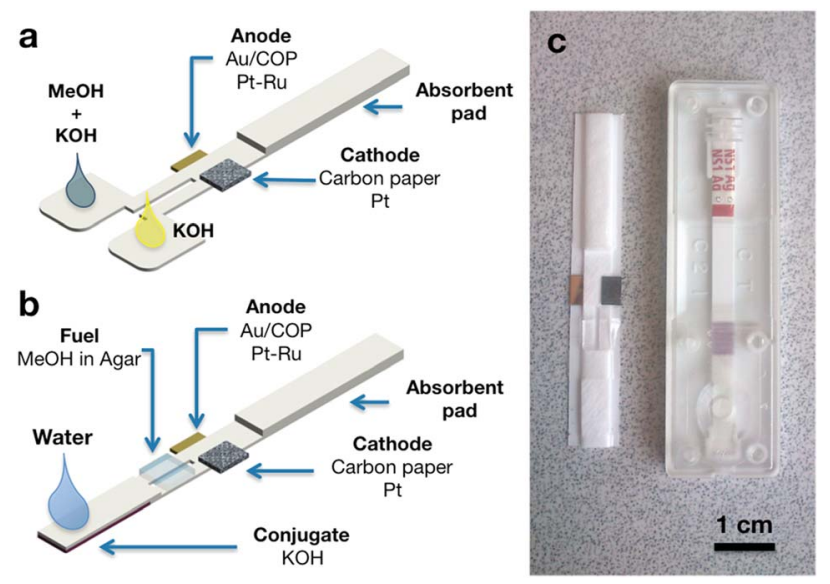

Fig. 2 (a) Schematic representation of a paper-based microfluidic fuel cell. (b) Concept of a lateral flow test strip fuel cell activated with water. (c) Comparison of a paper-based microfluidic fuel cell and commercial lateral flow test strip for dengue diagnosis. as an air-breathing cathode. ${ }^{\mathbf{1 6}}$ Catalyst layers were deposited by spray coating ink with a total metal loading of $1 \mathrm{mg} \mathrm{cm}^{-2}$ using $30 \mathrm{wt} \%$ PtRu on a Cabot Vulcan XC72, atomic ratio Pt : Ru $1: 1$ for the anode and Pt/C $40 \mathrm{wt} \%$ for the cathode (Johnson Matthey). As shown in Fig. 1, the anode was placed under the paper strip, while the cathode was attached on top to facilitate oxygen access from the atmosphere. The electrodes were placed with a separation of $1 \mathrm{~mm}$ from each other, so the electrode area in contact with the paper strip was $2 \times 5 \mathrm{~mm}$. In this configuration the fuel cell operates on the simultaneous addition of anolyte (methanol and $\mathrm{KOH}$ ) and catholyte $(\mathrm{KOH})$ in each of the sample pads. A test using color dyes to visualize the flows is shown in Fig. S1 and Video $1 . \dagger$ This configuration allowed the validation of the proposed fuel cell approach and was used to perform characterization under different operating conditions. However, this configuration would be difficult to use in a real application environment in which the lateral flow test is wetted with a single fluid (generally the fluid under analysis or sometimes fluid plus an additional buffer). The fuel cell design depicted in Fig. 2b brings the construction closer to conventional lateral flow strips as it only needs the application of one solution on the sample pad to operate. For comparison, Fig. 2c shows this device next to a commercial Rapid Dengue Test (Dengue NS1 AG, Standard Diagnostics Inc.). In this case, under the sample pad, we placed a conjugate that had been prepared by soaking a $5 \mathrm{~mm} \times 15 \mathrm{~mm}$ strip of the Fusion 5 membrane in a concentrated $\mathrm{KOH}$ solution $(10 \mathrm{M})$ and evaporating the water on a hot-plate at $140{ }^{\circ} \mathrm{C}$ for 5 minutes. In this design, flow separation in two parallel streams was achieved by cutting a $1 \times$ $8 \mathrm{~mm}$ slit in the membrane region between sample pad and fuel cell electrodes. Methanol was stored in a layer of agar gel cut in a $5 \mathrm{~mm} \times 5 \mathrm{~mm}$ square and placed over the slit, details of the preparation procedure are found in the ESI. $\uparrow$ The cathode side of the slit was laminated to prevent diffusion of methanol from the gel to the catholyte stream. For clarity, an exploded view of the device with its components can be found in Fig. S2. $\dagger$ When the device is fed with a small volume of water, $\mathrm{KOH}$ in the conjugate dissolves and flows towards the membrane. The flow is then diverted at the slit so the anode side receives methanol by diffusion from the agar gel. Thus $\mathrm{KOH}$ is present in the reaction zone in sufficient concentration throughout the membrane, maintaining ionic transport between the electrodes, whereas methanol is only flowing in the anode side and oxygen in the catholyte comes from air through the porous cathode. The sample continues to flow until it is completely absorbed by the absorbent pad. A visualization of these operations using color dyes is also given in the ESI. $\dagger$

\section{Results and discussion}

Before the characterization of the fuel cells, a preliminary electrochemical test was performed to study mass transport in paper devices by amperometry and show that the two parallel streams are effectively separated. Amperometry provides a direct measurement of fluid velocity through the measurement of diffusion-controlled currents. We have used it not only to monitor the diffusion across the anolyte-catholyte liquid interface, but 
also the duration of the signal given by a set volume of solution and in this way determine the average flow rate.

To do this, the Y-shape paper device was mounted on two sets of 3-gold band electrodes, with a separation of $1 \mathrm{~mm}$ between the sets. These were controlled using a multipotentiostat $(\mathrm{CH}$ Instruments 1030B), so that the working electrodes of the two cells could be polarized and measured independently. A volume of $250 \mu \mathrm{L}$ electrolyte solution $\left(0.5 \mathrm{M} \mathrm{KNO}_{3}\right)$ was added to one branch of the "Y" and, at the same time, an equal volume of a ferrocyanide solution $\left(2 \mathrm{mM} \mathrm{K} \mathrm{K}_{4} \mathrm{Fe}(\mathrm{CN})_{6}\right.$ in $\left.0.5 \mathrm{M} \mathrm{KNO}_{3}\right)$ was added to the other. These solutions then converged in the main section, where they flowed in parallel towards the absorbent. The current recorded by the working electrode placed under the ferrocyanide stream increased from zero until reaching a steady state that was maintained until the solution ran out. On the side of the stream with only electrolyte, the current remained close to zero throughout the experiment. The ESI $\uparrow$ contains more details of this experiment, which demonstrated that (i) the flow rate in this strip is around $80 \pm 5 \mu \mathrm{L} \mathrm{min}{ }^{-1}$, (ii) the solutions were not mixing inside the paper, and (iii) cross-diffusion at the interface between both streams was not significant at the position of the electrodes. The implication is that the two independent electrochemical reactions required in a microfluidic fuel cell can be performed in a single paper strip.

After that, the $I-V$ characteristic of the paper-based microfluidic fuel cells was tested at different concentrations of the fuel and $\mathrm{KOH}$ electrolyte. A Keithley 2400 Source-meter in galvanostatic mode together with a Keithley 2700 Multimeter controlled by using an in-house built LabView program allowed the recording of the fuel cell output voltage and the cathode potential vs. a $\mathrm{Ag} / \mathrm{AgCl}$ pseudo-reference electrode simultaneously. During these measurements, the Y-shaped device was used, so anolyte and catholyte were supplied continuously at each of the inlets and an additional wicking material (Kimwipe) was placed on the absorbent pad to maintain a steady flow rate
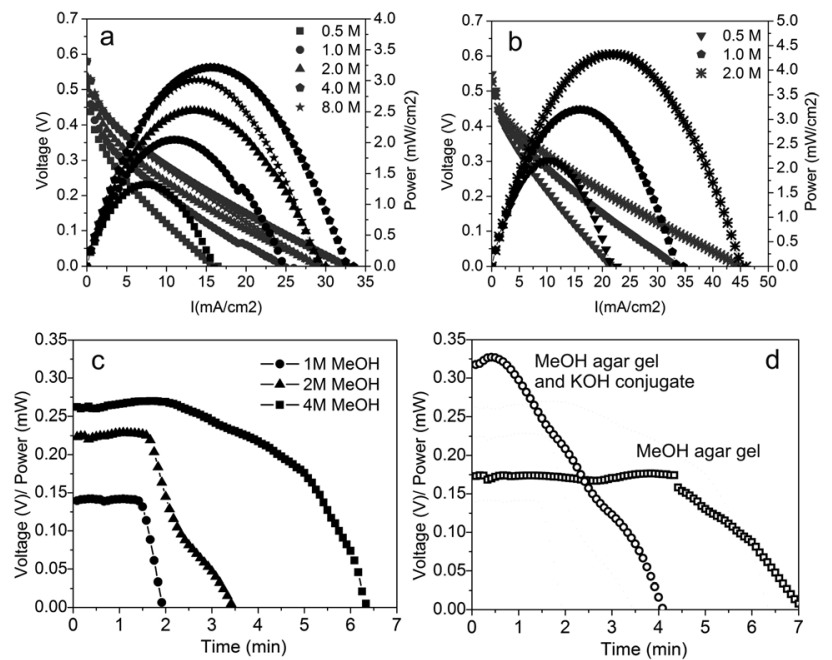

Fig. 3 Paper-based microfluidic fuel cell performance. (a) Polarization curves at different methanol concentrations in $1.0 \mathrm{M} \mathrm{KOH}$. (b) Polarization curves at different $\mathrm{KOH}$ concentrations using 4.0 M methanol. (c and d) Power output at a current load of $1 \mathrm{~mA}$. for times longer than 2 minutes. Fig. 3a shows the measured polarization curves for the fuel cell working at methanol concentrations ranging from $0.5 \mathrm{M}$ to $8.0 \mathrm{M}$, all in $1.0 \mathrm{M} \mathrm{KOH}$ solutions. The open circuit voltage observed under these conditions was $520 \pm 60 \mathrm{mV}$. A maximum power density of $3.2 \mathrm{~mW} \mathrm{~cm}^{-2}$ at a current density of $15.5 \mathrm{~mA} \mathrm{~cm}^{-2}$ was achieved with a methanol concentration of $4.0 \mathrm{M}$. Increasing the methanol concentration up to $8.0 \mathrm{M}$ did not increase the generated power. The reproducibility of the device performance can be seen in Fig. S6, $\uparrow$ showing a variation of less than $10 \%$ between devices, which can mainly be attributed to the variations when fixing the absorbent pad to the end of the strip. The measurements of the potentials in each electrode allow detection of the source of the main overpotentials in the cell (see Fig. S7†). It can be seen that both anode and cathode account for similar overpotentials when working at low methanol concentrations but that the cathode reaction is the major source of overpotential at higher methanol concentrations. Moreover, the absence of mass transport losses at increasing density currents no matter the methanol concentration suggests that the fuel cell internal resistance is one of the major sources of power loss. This is corroborated in Fig. 3b, which shows the polarization curves for the microfluidic fuel cell working with $\mathrm{KOH}$ concentrations of $0.5 \mathrm{M}, 1.0 \mathrm{M}$ and $2.0 \mathrm{M}$, all using a methanol concentration of $4.0 \mathrm{M}$. In this case, the maximum power density reached a value of $4.4 \mathrm{~mW} \mathrm{~cm}{ }^{-2}$ at a current density of $22.5 \mathrm{~mA} \mathrm{~cm}^{-2}$ using a $\mathrm{KOH}$ concentration of $2.0 \mathrm{M}$. These results show how the increase of electrolyte conductivity enhances the fuel cell performance. This is due to the reduction of cell resistance, which can also be achieved by geometrical design optimization (e.g. narrowing the distance between electrodes). The described characterization allowed us to set the optimal working point of our microfluidic fuel cells between 10 and $20 \mathrm{~mA} \mathrm{~cm}{ }^{-2}$ depending on the fuel and electrolyte concentration. After that, the Y-shaped devices were tested using only the finite absorbent pad at the end. In this configuration, they were supplied with $75 \mu \mathrm{L}$ anolyte solution ( $\mathrm{MeOH}$ and $\mathrm{KOH}$ ) and $75 \mu \mathrm{L}$ of catholyte solution (KOH) and subjected to a current load of $1 \mathrm{~mA}(10 \mathrm{~mA}$ $\mathrm{cm}^{-2}$ ). The evolution of the fuel cell output voltage was recorded for different methanol concentrations. Fig. 3c shows the evolution of the fuel cell output voltage (and power) where it can be seen that under all of the tested methanol concentrations, the fuel cell sustained a steady voltage output for at least 2 minutes, which corresponds to the time required for the reactants to flow from the sample pads to the absorbent pad. After this, the behavior of the output voltage depends on the methanol concentration flowing in the anolyte. At low concentrations (1.0-2.0 M) the voltage drops steeply shortly after the reactants have stopped to flow, whereas at a methanol concentration of $4.0 \mathrm{M}$ the signal starts to wane for about 3 additional minutes until it finally drops to zero. This is due to the contribution of the methanol diffusion. Finally, we tested the lateral-flow like fuel cell. First, the device was mounted with the methanol gel on top of the entry channel and the fuel cell was supplied with a single shot of $150 \mu \mathrm{L}$ of $1.0 \mathrm{M} \mathrm{KOH}$ solution. This allowed us to test if the methanol delivered by the gel was sufficient to maintain a constant voltage as long as the electrolyte flowed. 
As it can be seen in Fig. 3d, the fuel cell yielded a constant output voltage for about 4.5 minutes - long after the capillary flow had stopped. This was probably due to the fact that the gel was able to supply methanol to the anode stream at a concentration over $8.0 \mathrm{M}$, which also would explain why the voltage magnitude was almost $0.1 \mathrm{~V}$ lower than the one previously obtained at $4.0 \mathrm{M}$. The fuel cell power output was again tested by placing a $\mathrm{KOH}$ conjugate under the sample pad and the methanol gel over the anode stream. In this case, a volume of $150 \mu \mathrm{L}$ of D.I. water was required to start the device. Fig. 3d shows that the resulting output voltage rose to $330 \mathrm{mV}$ for over a minute and then declined steadily for another three minutes until it suddenly dropped to zero. This response is due to the inhomogeneous dissolution of $\mathrm{KOH}$ into the water stream leading to a changing $\mathrm{KOH}$ concentration over time. At the beginning, a large amount of $\mathrm{KOH}$ is dragged by the water, resulting in an initial power value of $0.32 \mathrm{~mW}$. As the $\mathrm{KOH}$ in the conjugate dissolves and its concentration falls, the voltage of the fuel cell drops accordingly. The dissolution of $\mathrm{KOH}$ within the strip and the $\mathrm{pH}$ evolution of the incoming water flow are presented in Fig. S4 and Video $3 . \dagger$

The results presented here clearly show the potential of implementing a microfluidic fuel cell in a paper platform. The flow driven by capillarity is enough to ensure an effective separation of reactants in two parallel streams and yield power performances comparable to pump-assisted microfluidic fuel cells reported in the literature (Table S1†). Nevertheless, it is worth noticing that the pumping systems required for all of the previously reported microfluidic fuel cells might represent a significant power consumption, which can sometimes exceed the amount of power delivered by the fuel cell itself.

Regarding the performance under a current load, the fuel cell is able to provide a continuous power of more than $2.0 \mathrm{~mW}$ $\mathrm{cm}^{-2}$ for 4.2 minutes when fed with $150 \mu \mathrm{L}$ of separated reactants or $1.75 \mathrm{~mW} \mathrm{~cm}^{-2}$ for about 4.5 minutes when fed with a single electrolyte and a methanol gel. In both cases, the power density obtained exceeds that of current printed batteries, ${ }^{17}$ which clearly shows the potential of this approach. The faradic efficiency of the paper fuel cell was calculated from the constant current curves, yielding a value of $c a$. 1\% due to low fuel utilization. This figure evidences that there is still plenty of room for optimization. In this respect, some strategies to increase the fuel cell efficiency may come from enhancing fuel utilization, e.g. tuning flow rate by changing the paper type, channel geometry or absorbent material; or improving the electrode arrangement and using a higher catalyst load. ${ }^{18}$ Alternatively, the incorporation of other catalytic materials (preferably base metals or enzymatic catalysts) would allow the use of a diversity of fuels and electrolytes in both acidic and alkaline media. These compounds could also be stored within the same porous matrices and released upon the addition of the liquid sample. ${ }^{19}$

It is worth noticing that despite being proof-of-concept prototypes, the power yielded by these lateral flow-based fuel cells meet the power needs of the two most popular rapid tests in the market, namely glucometers and electronic pregnancy tests. Amperometric glucometers like the one described by Microchip Technology ${ }^{20}$ use a $3.0 \mathrm{~V}$ button-cell battery to power an electronics module that correlates the measured electrical current with the glucose concentration in a drop of blood and display the result within a few seconds. During its operation, the device requires a current of $1.1 \mathrm{~mA}$. On the other hand, Clearblue digital pregnancy tests ${ }^{3}$ are able to estimate the number of pregnancy weeks by measuring the intensity of a lateral flow test line with a disposable optical reader. This reader is also powered using a $3.0 \mathrm{~V}$ button-cell battery. Although the total time to run one of these tests is around 3 minutes, the optical measurement lasts only a couple of seconds and has a maximum current consumption of $4 \mathrm{~mA}$. These examples show the potential of our devices to fulfill the power demands of portable point-of-care devices. It is also clear that fuel cell performance may be improved to meet the requirements of specific applications while maintaining the size constrain of standard lateral flow test strips. Moreover, it has already been demonstrated that using ultra low power electronics, the voltage output of a single fuel cell can be ramped up from values as low as $0.13 \mathrm{~V}$ up to $5.5 \mathrm{~V}$, which is adequate to power electronic modules, sensors and indicators. ${ }^{21,22}$ Nevertheless, further optimization of the presented approach could lead to the implementation of fuel cell stacks, which would directly increase the output voltage of the device without further conversion stages.

\section{Conclusions}

We have presented the first paper-based methanol microfluidic fuel cell. One of the most important advantages of the devices presented here is that the use of porous membranes eliminates the need of external pumps because reactants flow by capillary forces. We consider that this approach overcomes the miniaturization difficulties associated with the use of ancillary devices in microfluidic fuel cells and places them in an application niche where they probably fulfill future energy requirements. This is reinforced by the fact that all the power produced by the fuel cell is actually available for its use and that its operation requires no effort by the user.

We have shown that standard paper and fiber-based materials can be used to effectively perform the different functions required for a microfluidic fuel cell. It was demonstrated that the same material could perform as channel, reagent storage, or waste sink depending on its shape and arrangement. Other microfluidic operations such as accurately dividing a stream in two separate but adjacent flows can be achieved simply by slitting the paper in the right manner and place.

The design of the presented fuel cells has been inspired on typical lateral flow test strips to enable a more straightforward integration and cost-effective manufacturing. Here we have shown the feasibility of storing both the $\mathrm{KOH}$ electrolyte and methanol fuel within the paper strip, so that the fuel cell works on the addition of water. In practice, the fuel cell can generate power when adding any liquid as long as it provides a continuous flow for a certain period of time. Integrated within a lateral flow test, the fuel cell can clearly be used to enable new onboard functions. For instance, heating up a sample to speed up chemical reactions and even powering a small chip that enables 
quantification of analytes are two valuable possibilities. Ultimately, the unique electrochemical features of fuel cells make them capable of generating the power needed to quantify the results of a rapid flow test from the same sample that is analyzed (e.g. glucose in blood ${ }^{23}$ or urea in urine ${ }^{24}$ ). We foresee that combining the advantages of paper microfluidics with the emerging technology of printed and organic electronics would lead to the development of autonomous, portable, environmentally friendly, disposable diagnostics devices. Ideally, the electrodes and conductive tracks would be printed directly on the plastic and paper substrates and the devices would be assembled in the same roll-to-roll process.

\section{Acknowledgements}

This research was partially supported by a Marie Curie International Outgoing Fellowship within the 7th European Community Framework Programme and DURSI of the Generalitat de Catalunya under contract 2009SGR00228. N. S. would like to thank the financial support received from her postdoctoral program Ramón y Cajal. S. R. acknowledges project ENE2010-15381 for financial support. The authors acknowledge the support for the publication fee by the CSIC Open Access Publication Support Initiative through its Unit of Information Resources for Research (URICI).

\section{References}

1 A. K. Yetisen, M. S. Akram and C. R. Lowe, Lab Chip, 2013, 13, 2210-2251.

2 S. Vashist, O. Mudanyali, E. M. Schneider, R. Zengerle and A. Ozcan, Anal. Bioanal. Chem., 2013, 1-15.

3 http://www.clearblue.com, Geneva, Switzerland, 2008-2012.

4 A. P. F. Turner, Chem. Soc. Rev., 2013, 42, 3184-3196.

5 L. Nyholm, G. Nyström, A. Mihranyan and M. Strømme, Adv. Mater., 2011, 23, 3751-3769.

6 N. K. Thom, K. Yeung, M. B. Pillion and S. T. Phillips, Lab Chip, 2012, 12, 1768-1770.

7 L. Zhang, M. Zhou, D. Wen, L. Bai, B. Lou and S. Dong, Biosens. Bioelectron., 2012, 35, 155-159.
8 G. P. M. K. Ciniciato, C. Lau, A. Cochrane, S. S. Sibbett, E. R. Gonzalez and P. Atanassov, Electrochim. Acta, 2012, 82, 208-213.

9 T. H. Nguyen, A. Fraiwan and S. Choi, Biosens. Bioelectron., 2014, 54, 640-649.

10 E. Kjeang, N. Djilali and D. Sinton, J. Power Sources, 2009, 186, 353-369.

11 R. Ferrigno, A. D. Stroock, T. D. Clark, M. Mayer and G. M. Whitesides, J. Am. Chem. Soc., 2002, 124, 12930-12931.

12 S. A. Mousavi Shaegh, N.-T. Nguyen and S. H. Chan, Int. J. Hydrogen Energy, 2011, 36, 5675-5694.

13 J. L. Osborn, B. Lutz, E. Fu, P. Kauffman, D. Y. Stevens and P. Yager, Lab Chip, 2010, 10, 2659-2665.

14 M. J. Gonzalez-Guerrero, J. P. Esquivel, D. Sanchez-Molas, P. Godignon, F. X. Munoz, F. J. del Campo, F. Giroud, S. D. Minteer and N. Sabate, Lab Chip, 2013, 13, 2972-2979.

15 E. R. Choban, J. S. Spendelow, L. Gancs, A. Wieckowski and P. J. A. Kenis, Electrochim. Acta, 2005, 50, 5390-5398.

16 R. S. Jayashree, L. Gancs, E. R. Choban, A. Primak, D. Natarajan, L. J. Markoski and P. J. A. Kenis, J. Am. Chem. Soc., 2005, 127, 16758-16759.

17 http://www.enfucell.com/, Vantaa, Finland, 2013.

18 M. R. Thorson, F. R. Brushett, C. J. Timberg and P. J. A. Kenis, J. Power Sources, 2012, 218, 28-33.

19 B. Lutz, T. Liang, E. Fu, S. Ramachandran, P. Kauffman and P. Yager, Lab Chip, 2013, 13, 2840-2847.

20 N. Dalvi, Glucose Meter Reference Design, Microchip Technology Inc., 2013, http://ww1.microchip.com/ downloads/en/DeviceDoc/00001560A.pdf.

21 A. Zebda, S. Cosnier, J. P. Alcaraz, M. Holzinger, A. Le Goff, C. Gondran, F. Boucher, F. Giroud, K. Gorgy, H. Lamraoui and P. Cinquin, Sci. Rep., 2013, 3, 1516.

22 J. P. Esquivel, J. Colomer-Farrarons, M. Castellarnau, M. Salleras, F. J. del Campo, J. Samitier, P. Miribel-Catala and N. Sabate, Lab Chip, 2012, 12, 4232-4235.

23 F. M. Cuevas-Muñiz, M. Guerra-Balcázar, J. P. Esquivel, N. Sabaté, L. G. Arriaga and J. Ledesma-García, J. Power Sources, 2012, 216, 297-303.

24 R. Lan, S. Tao and J. T. S. Irvine, Energy Environ. Sci., 2010, 3, 438-441. 